\title{
Inhibition of neutral sphingomyelinases in skeletal muscle attenuates fatty-acid induced defects in metabolism and stress
}

\author{
Mahesh Kumar Verma, Aggunda Nagaraju Yateesh, Korrapati Neelima, Niketa Pawar, Kandoor Sandhya, \\ Jayaram Poornima, Mudigere N Lakshmi, Sivakumaran Yogeshwari, Puttrevana M Pallavi, Anup M Oommen, \\ Baggavalli P Somesh and Madanahalli R Jagannath*
}

\begin{abstract}
Background: Chronic metabolic overload leads to insulin resistance in a variety of tissues. It has been shown that exposure to saturated fatty acid palmitate can cause insulin resistance in skeletal muscle cells. Fatty acid induced synthesis of ceramide is considered to be one of the major causes for insulin resistance. Both de novo synthesis and sphingomyelin hydrolysis by sphingomyelinase are implicated for ceramide generation. Aim of this study was to evaluate the impact of neutral sphingomyelinase (nSMase) inhibition on saturated fatty acid induced lipotoxicity and insulin resistance in skeletal muscle myotubes.

Results: Treatment of saturated fatty acid (palmitate) but not unsaturated fatty acid (oleate) caused an up-regulation in expression of various nSMase genes which are associated with ceramide synthesis through the salvage pathway. Inhibition of nSMase by a pharmacological inhibitor (GW4869) partially reverted the palmitate induced insulin resistance in C2C12 myotubes. Inhibition of nSMase improved metabolic functions of myotubes as measured by improved oxidative capacity in terms of increased mitochondrial number, PGC1a expression and ATP levels with concomitant decrease in intramyocellular triglyceride levels. Palmitate induced inflammatory response was also reduced by nSMase inhibitor. GW4869 treatment reduced palmitate induced oxidative and endoplasmic reticulum stress and improved cell survival.
\end{abstract}

Conclusion: In this study, we provide evidences that inhibition of nSMase can protect skeletal muscles from saturated fatty acid induced insulin resistance, metabolic dysfunction, cellular stress and inflammation.

Keywords: Sphingomyelinase; Ceramide; C2C12 myotubes; Insulin resistance; Oxidative capacity; Cellular stress; T2DM

\section{Background}

Insulin resistance is a central phenomenon under metabolic disorders such as type 2 diabetes mellitus (T2DM). Understanding the defects in metabolism and underlying regulatory molecular network is of importance to design an effective therapy to manage T2DM and peripheral insulin resistance. Dealing with insulin resistance in skeletal muscle is of prime importance because this tissue accounts for as high as $95 \%$ glucose disposal during hyperglycemic hyperinsulinemic condition (Baron et al.

\footnotetext{
* Correspondence: m.r.jagannath@connexios.com
Connexios Life Sciences Private Ltd., No. 49, First Main road, 3rd phase, JP

* Correspondence: m.r.jagannath@connexios.com
Connexios Life Sciences Private Ltd., No. 49, First Main road, 3rd phase, JP Nagar, Bangalore 560 078, India
}

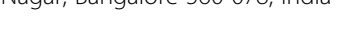

(van der Vusse and Reneman 1996).

Skeletal muscles from diabetic patients show impaired glucose uptake, storage and utilization because of diminished insulin signaling (Kelley et al. 1996; Kim et al. 1999; Krook et al. 2000; Bouzakri et al. 2003). Insulin resistance mediated defects are not limited only to glucose metabolism instead can influence several aspects of metabolism like overall reduction in the oxidative capacity as evident by the number/activity of mitochondria and reduced ATP levels (Simoneau and Kelley 1997; Kelley et al. 2002; Scheuermann-Freestone et al. 2003; Schrauwen and Hesselink 2004; Asmann et al. 2006; Szendroedi et al. 
2007). These impairments are also accompanied by increased oxidative and endoplasmic reticulum (ER) stress and chronic low grade inflammation (Hey-Mogensen et al. 2010).

One of the major factors causing insulin resistance is elevated plasma free fatty acids (FFA) levels (Itani et al. 2002). In fact, increased rate of lipolysis from adipose tissue and VLDL secretion from liver are observed in T2DM patients and both of these might contribute for muscle FA overload. Further, augmentation in muscle fat storage is contributed by raised plasma FFA level, increased fat uptake (Bonen et al. 2004), and defective or incomplete oxidation in skeletal muscle of diabetic patients (Mootha et al. 2003; Patti et al. 2003; Mensink et al. 2007; Mogensen et al. 2007; Koves et al. 2008). Fatty acid overload leads to its accumulation in skeletal muscle mainly in form of diacylglycerol (DAG), triglyceride (TG) or ceramide in obese and T2DM patients (Goodpaster et al. 2000; Bonen et al. 2004). Increased fat storage in form of TG can be directly correlated to insulin resistance in the sedentary subjects (McGarry 2002) but not in the exercise trained ones (Goodpaster et al. 2001). On the other hand, storage of fat in terms of neutral TG might be a protective mechanism (Listenberger et al. 2003). This hypothesis is strengthened by studies which showed that unsaturated fatty acid oleate, which is a preferred substrate for TG synthesis, did not demonstrate toxic impacts (Listenberger et al. 2003; Yuzefovych et al. 2010). These evidences lead to the speculation that lipid metabolism in the form other than TG storage is required for development of insulin resistance.

Metabolism of lipid into complex lipid such as ceramide is reported to develop insulin resistance. Ceramide levels in cells are controlled by de novo synthesis or by its generation through salvage pathway using sphingomyelinase (SMase). The enzyme serine palmitoyl transferase (SPT) catalyzes the rate limiting step for de novo sphingolipid synthesis. Its expression and activity are increased in T2DM, insulin resistant subjects, and in response to saturated fatty acid and inflammatory cytokines. A number of studies indicated that pharmalogical inhibition of SPT can protect from insulin resistance and lipotoxicity (Holland et al. 2007; Ussher et al. 2010). The de novo synthesis of ceramides is triggered primarily from saturated fatty acids, which are one of the major contributors for insulin resistance, under various conditions relevant to metabolic syndrome such as lipotoxicity, inflammation, glucocorticoids (Holland et al. 2007; Zierath 2007; Blachnio-Zabielska et al. 2010). Treatment of cells with ceramide itself can cause insulin resistance (SchmitzPeiffer et al. 1999; Pickersgill et al. 2007). Increased ceramide levels lead to inflammation, ER stress, oxidative stress and apoptosis (Turpin et al. 2006; de Mello et al. 2009). In obese T2DM patients, increased ceramide level is observed in plasma (Haus et al. 2009), liver (Boon et al. 2013), adipose (Blachnio-Zabielska et al. 2012) and skeletal muscle (Adams et al. 2004). Moreover, skeletal muscle ceramide levels are found to be increased in subjects at risk of development of T2DM (Straczkowski et al. 2007). In fact, there exists a positive correlation between ceramide concentration and degree of insulin resistance (Straczkowski et al. 2004, 2007; Turpin et al. 2006; Haus et al. 2009; Blachnio-Zabielska et al. 2012). Therefore, it was inferred that fatty acid overload induced lipotoxicity and insulin resistance are mediated, at least in part, by synthesis of ceramide from saturated fatty acids while inhibition of ceramide synthesis could protect both cultured cells (Schmitz-Peiffer et al. 1999; Turpin et al. 2006; Pickersgill et al. 2007) and rodent model of disease (Holland et al. 2007; Yang et al. 2009; Ussher et al. 2010).

Ceramide and sphingomyelin are interconverted by enzymes sphingomyeline synthase (SMS) and sphingomyelinase (SMase). Sphingomyelin to ceramide ratio is inversely correlated to neutral SMase (nSMase) level indicating important role of nSMase in maintaining levels of these sphingolipids (Dobrzyń et al. 2002). Inflammatory cytokines can increase nSMase activity thereby increasing ceramide levels (Long and Pekala 1996). Level of nSMase is found to be increased in impaired glucose tolerant subjects (Straczkowski et al. 2007) and high fat diet induced diabetic rodents (Murase et al. 1998; Shah et al. 2008). Recent review from our laboratory exploring the gene expression profiles revealed that in T2DM patients the levels of sphingomyelinase are up-regulated (Pralhada Rao et al. 2013). Conversely, level of nSMase is decreased after exercise (Dobrzyń et al. 2002; Helge et al. 2004) leading to an increase in sphingomyelin to ceramide ratio. These reports suggest that targeting nSMase can reduce ceramide level thereby making tissue insulin sensitive. Despite these indications, there is no substantial data available in literature which can demonstrate the role of nSMase for skeletal muscle insulin resistance.

Here we report that inhibition of membrane localized neutral sphingomyelinase by a small molecule inhibitor can protect $\mathrm{C} 2 \mathrm{C} 12$ skeletal muscle myotubes from palmitate induced insulin resistance. We demonstrate that nSMase expression is increased during palmitate treatment but not during oleate treatment. Inhibition of nSMase improves myotubes oxidative capacity and reduces triglyceride storage. Its inhibition also reduces cellular stress, inflammation and improves cell survival. Therefore, we conclude that nSMase inhibition can protect skeletal muscle from saturated FFA overload induced lipotoxic impacts. Hence, we believe that this strategy hold promise to provide opportunity for therapeutic interventions to treat muscle pathology present in metabolic syndrome. 


\section{Results}

Palmitate treatment increases nSMase expression in C2C12 myotubes

Following palmitate $(750 \mu \mathrm{M})$ or oleate $(750 \mu \mathrm{M})$ treatment to differentiated myotubes for $16 \mathrm{hrs}$, we measured expression of nSMase genes isoforms. Expression of nSMase- 1 was up-regulated by 1.38 fold under palmitate treatment $(\mathrm{P}<0.01$, Figure $1 \mathrm{~A})$ when compared to vehicle control set. An increase in nSMase-2 expression was also observed under palmitate condition (1.85 fold of vehicle control, $\mathrm{P}<0.05$, Figure $1 \mathrm{~B}$ ) whereas nSMase- 3 expression was slightly increased (1.29 fold of vehicle control, $\mathrm{P}<0.05$, Figure $1 \mathrm{C}$ ). Oleate treatment was unable to induce the expression of nSMaes (Figure 1A, B, C). These results indicate an increase in nSMase expression after exposure to saturated FFA.

We next measured the expression of SPT (SPTLC1), a rate limiting enzyme in the de novo pathway of ceramide biosynthesis. Similar to an increase in nSMase expression, SPTLC1 expression was also up-regulated under palmitate treatment (1.22 fold of vehicle control, $\mathrm{P}<0.05$ ) but not under oleate treatment (Figure 1D). Thus palmitate treatment can increase both de novo and nSMase mediated ceramide synthesis pathways indicating a potential increase in ceramide synthesis. However, we did not measure total ceramide levels in the cells but studied functional outcomes as mentioned henceforth.

\section{Inhibition of nSMase reduces palmitate induced insulin resistance in C2C12 myotubes}

We hypothesized that nSMase inhibition can reduce skeletal muscle insulin resistance caused by palmitate overload. $\mathrm{C} 2 \mathrm{C} 12$ myotubes were treated with palmitate in presence or absence of nSMase inhibitor, GW4869 $(10 \mu \mathrm{M})$ for $16 \mathrm{hrs}$. After $16 \mathrm{hrs}$, myotubes were treated with or without insulin for $10 \mathrm{~min}$ and insulin sensitivity was assessed by phosphorylated Akt. As seen in Figure 2A, insulin treatment could increase Akt phosphorylation in myotubes treated with vehicle control $(\mathrm{P}<0.001)$. Palmitate treatment showed reduced Akt phosphorylation in response to insulin $(\mathrm{P}<0.01)$ hence indicated development of insulin resistance. Inhibition of nSMase with palmitate treatment maintained Akt phosphorylation $(\mathrm{P}<0.01)$ and thus improved insulin sensitivity (Figure 2A).

We next studied insulin induced changes in PDK4 expression, a gene whose expression level is negatively regulated with glucose oxidation. The levels of PDK4 are known to reduce with insulin treatment. Expression of PDK4 was up-regulated by palmitate exposure (Figure 2B). Insulin treatment could reduce its expression only in vehicle treated cells $(0.75$ fold, $\mathrm{P}<0.01)$ but not in palmitate treated cells thus demonstrating progression of insulin resistance (Figure 2B). Inhibition of nSMase in this condition maintained insulin sensitivity of skeletal muscle cells as insulin was able to inhibit PDK4 expression $(\mathrm{P}<0.01$, Figure $2 \mathrm{~B})$. Hence we conclude that
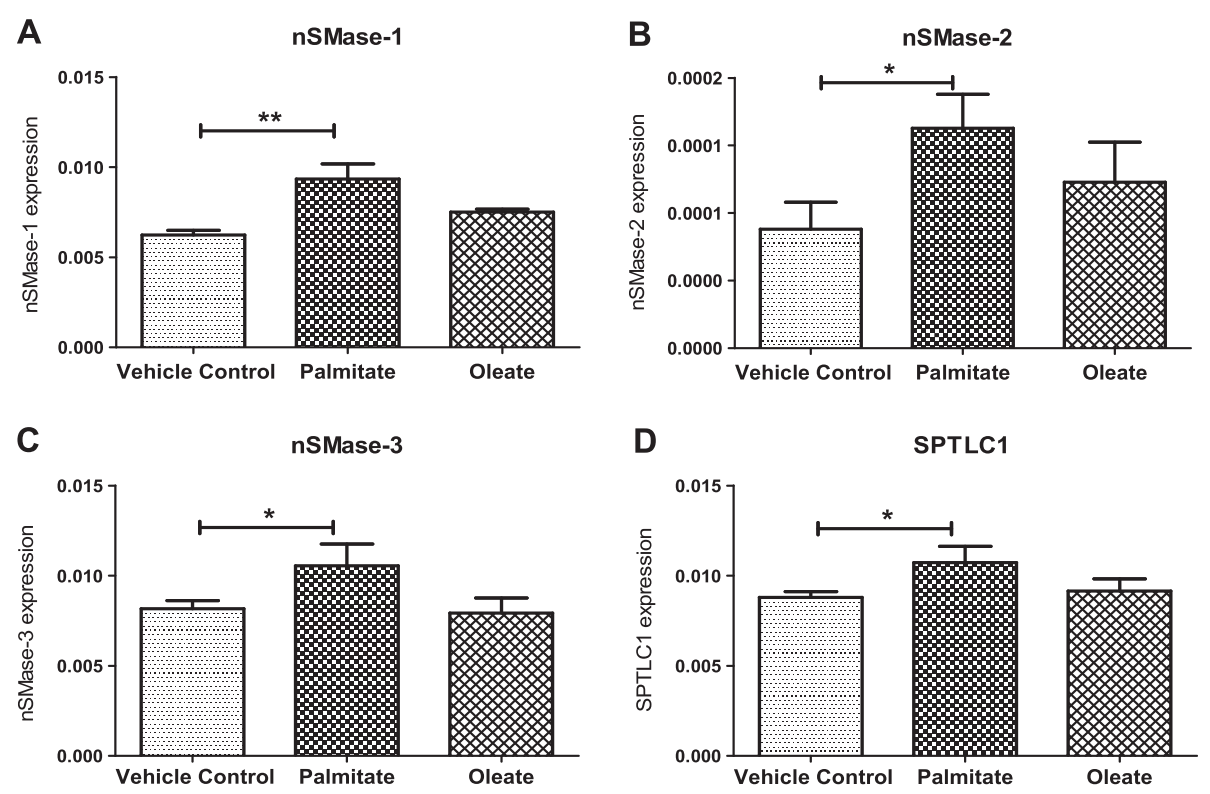

Figure 1 Palmitate treatment increases nSMases expression. Differentiated $\mathrm{C} 2 \mathrm{C} 12$ myotubes were treated with vehicle control or palmitate or oleate $(750 \mu \mathrm{M})$ for $16 \mathrm{hrs}$ as described under 'Methods' section. After treatments, total RNA was isolated and mRNA levels of nSMase-1 (A), nSMase-2 (B), nSMase-3 (C) and SPTLC1 (D) were quantified by quantitative real time PCR using $\beta$-actin as housekeeping gene control. Data are presented as mean + standard deviation. $n=4,{ }^{*} P<0.01,{ }^{*} P<0.05$, one way ANOVA with Newman-Keuls post test was performed for statistical analyses. 
A

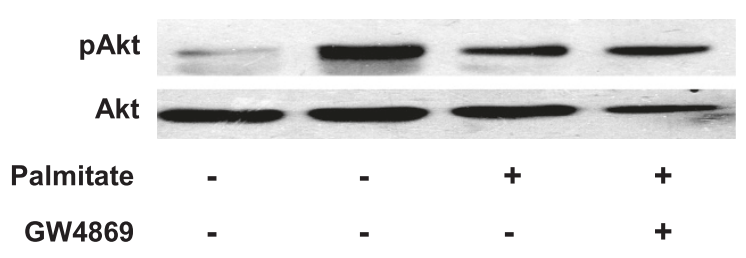

Akt Phosphorylation
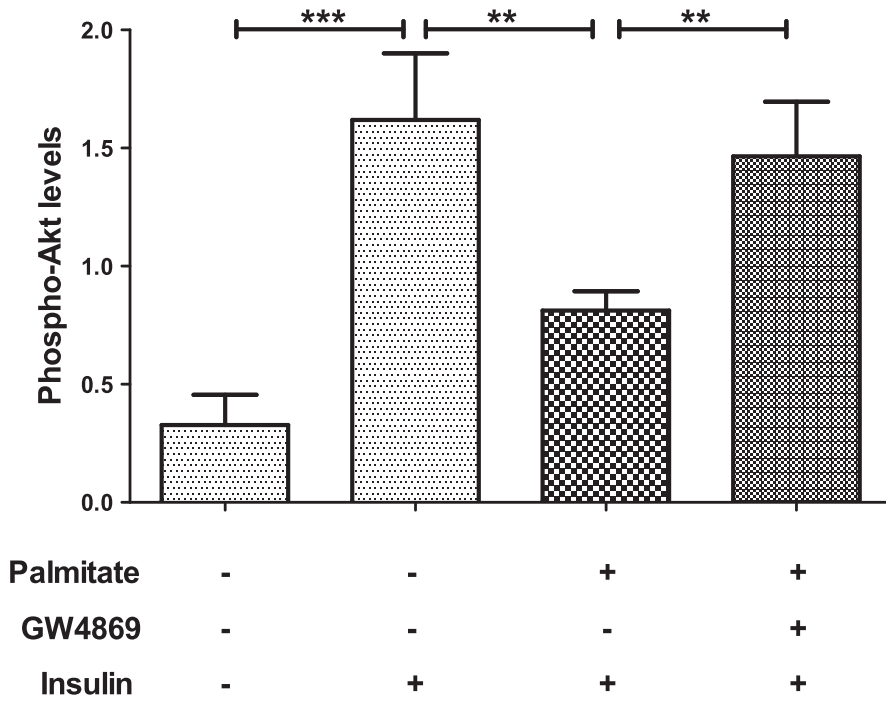

\section{B}

PDK4

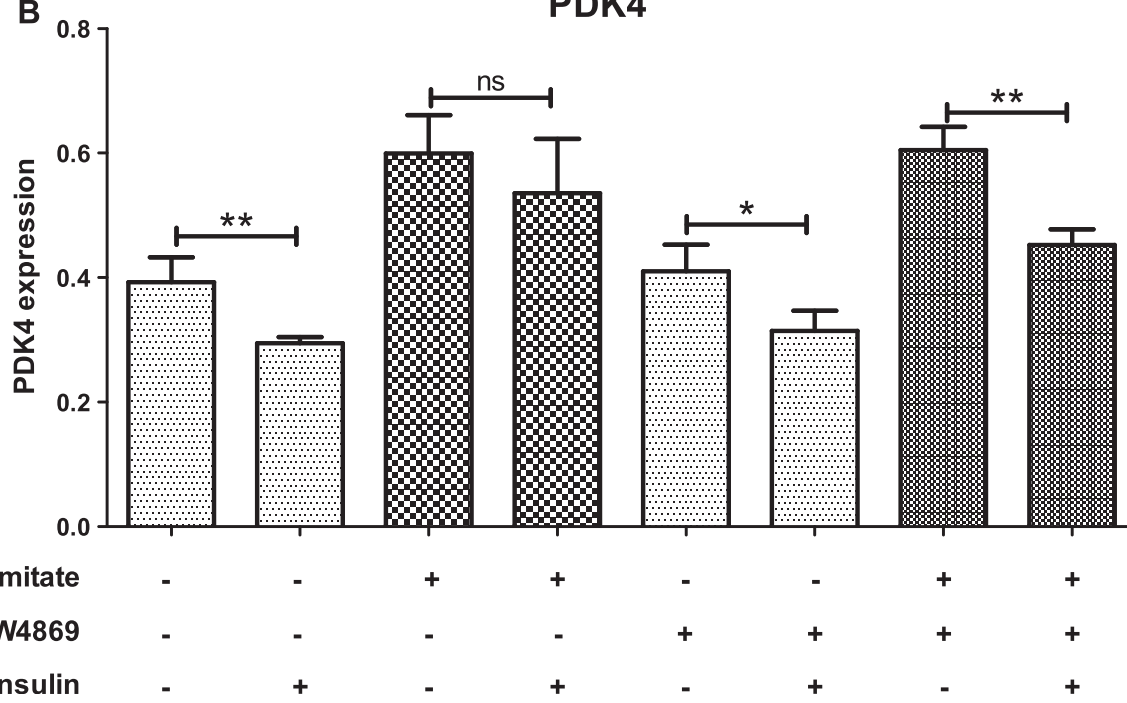

Figure 2 Palmitate induced insulin resistance is alleviated by nSMase inhibition. (A) After palmitate treatment, C2C12 myotubes were starved for $30 \mathrm{~min}$. Myotubes were then treated with or without insulin for $10 \mathrm{~min}$ followed by lysing in lysis buffer. Insulin induced Akt phosphorylation was measured in total cell lysate by Western blotting. Unphosphorylated levels of Akt were also measured as control for phosphorylation (Upper panel). Lower panel: quantification of Akt phosphorylation $\left(n=6,{ }^{* *} \mathrm{P}<0.001,{ }^{* *} \mathrm{P}<0.01\right)$ (B) $\mathrm{C} 2 \mathrm{C} 12$ cells were treated with or without palmitate for 2 hrs in presence or absence of nSMase inhibitor (GW4869). Insulin was also present in the treatments for indicated sets. Gene expression level of PDK4 was analyzed by quantitative real time PCR using $\beta$-actin as housekeeping gene control. Data are presented as mean + standard deviation. $n=4$, ${ }^{*} \mathrm{P}<0.01,{ }^{*} \mathrm{P}<0.05$, ns: not significant, one way ANOVA with Newman-Keuls post test was performed for statistical analyses. 
nSMase inhibition protects skeletal muscle from palmitate induced insulin resistance. Oleate treatment increased PDK4 expression in $\mathrm{C} 2 \mathrm{C} 12$ cells, it did not cause severe insulin resistance as insulin treatment was able to inhibit its expression $(\mathrm{P}<0.01$, Additional file 1$)$. Inhibition of nSMase maintained insulin sensitivity of oleate treated cells.

\section{Inhibition of nSMase results in improved oxidative capacity and metabolism}

Since insulin resistance is associated with impaired metabolism, we asked whether nSMase inhibition can improve metabolic functions of skeletal muscles. We measured impact of nSMase inhibition on expression of PGC1 $\alpha$, a gene which is implicated for mitochondrial biogenesis, fat oxidation and energy metabolism. Exposure of palmitate reduced PGC1 $\alpha$ expression to 0.47 fold when compared to vehicle control $(\mathrm{P}<0.01$, Figure $3 \mathrm{~A})$. Inhibition of nSMase in this background increased PGC1 $\alpha$ expression by $30 \%$ $(\mathrm{P}<0.05$, Figure 3A). Mitochondrial DNA copy number, a surrogate measurement for mitochondria number, was reduced by palmitate treatment $(0.39$ fold of vehicle control, $\mathrm{P}<0.05)$ and nSMase inhibition could restore the mitochondrial number $(\mathrm{P}<0.05$, Figure $3 \mathrm{~B})$. In consistence with this, cellular ATP levels were found to be reduced under palmitate treatment $(0.41$ nano-moles $/ \mathrm{mg}$ versus 0.72 nano-moles/mg under vehicle control, $\mathrm{P}<0.01)$ and nSMase inhibitor increased its level by $35 \%$ ( 0.56 nano- moles/mg, $\mathrm{P}<0.05$, Figure $3 \mathrm{C}$ ). Taken together, nSMase inhibition improved oxidative capacity of skeletal muscle myotubes.

We reasoned that improved insulin sensitivity and oxidative capacity by nSMase inhibition would impact fat storage. Under palmitate treatment, triglyceride storage was enhanced by 3.65 fold $(50 \mu \mathrm{g} / \mathrm{mg}$ versus $14 \mu \mathrm{g} / \mathrm{mg}$ under vehicle control, $\mathrm{P}<0.001$, Figure $3 \mathrm{D})$. Inhibition of nSMase under palmitate treatment led to a partial reduction in cellular triglyceride storage $(41 \mu \mathrm{g} / \mathrm{mg}, \mathrm{P}<0.01$, Figure $3 \mathrm{C}$ ). Hence, nSMase inhibition improves overall metabolic function of skeletal muscle.

\section{Neutral SMase inhibition reduces inflammation and cellular stress}

We next examined the impact of nSMase inhibition on cellular health. Palmitate treatment enhanced JNK phosphorylation $(2.02$ fold of control, $\mathrm{P}<0.05)$ and $\mathrm{NF}_{\mathrm{K}} \mathrm{B}$ activation as measured by reduction in $\mathrm{I}_{\kappa} \mathrm{B}$ levels $(0.44$ fold of vehicle control, $\mathrm{P}<0.01$, Figure $4 \mathrm{~A}$ ) thus indicating enhanced stress signaling. Inhibition of nSMase under this background reduced stress signaling as evident by reduced JNK phosphorylation (1.07 fold of vehicle control, $\mathrm{P}<0.01)$ and restoration of $\mathrm{I} \kappa \mathrm{B}$ levels $(0.90$ fold, $\mathrm{P}<0.01$, Figure 4A). In consistence with these data, palmitate induced increase in the IL6 expression (16 fold of control, $\mathrm{P}<0.001)$ was partially decreased by nSMase inhibition $(P<0.001$, Figure $4 B)$. Treatment of myotubes
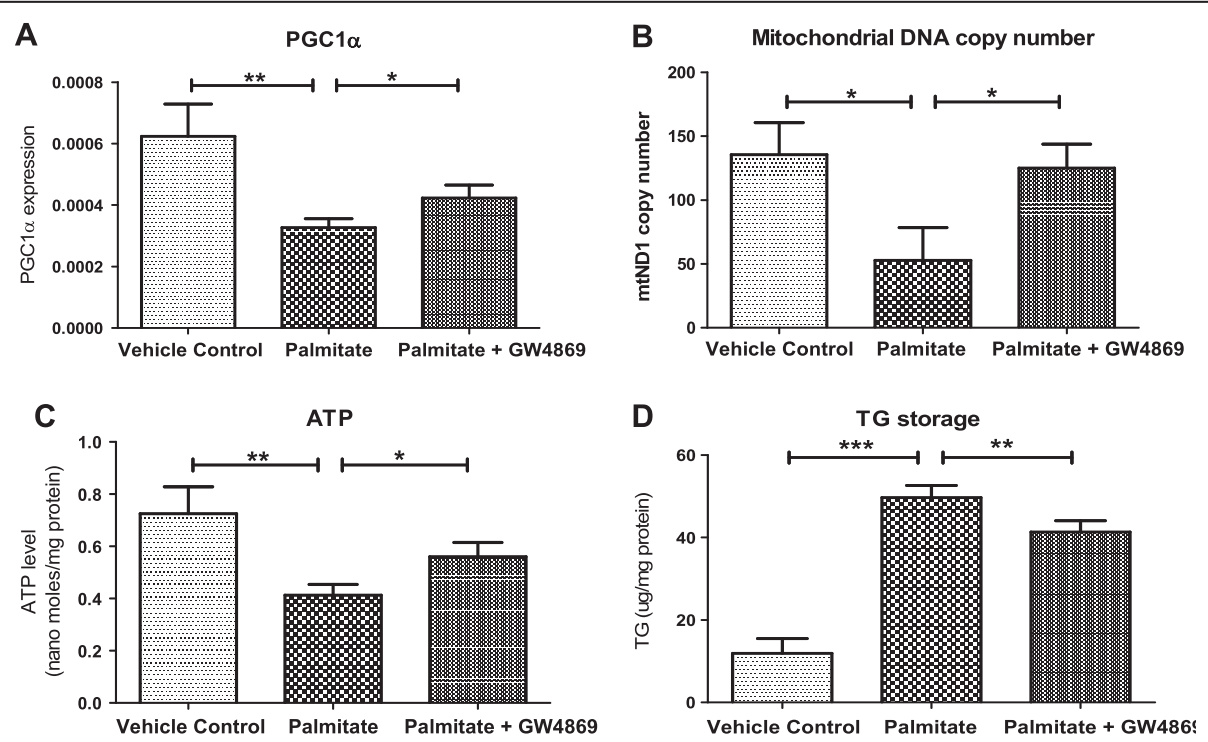

Figure 3 Inhibition of nSMase improves oxidative capacity of C2C12 myotubes and reduces TG storage. (A) Analysis of PGC1a gene expression in C2C12 myotubes cultured under palmitate condition in presence or absence of nSMase inhibitor (GW4869). $\beta$-actin was used as housekeeping gene control. (B) Mitochondrial DNA copy numbers were estimated by quantitative real time PCR using total DNA isolated from myotubes cultured under control or palmitate condition in presence or absence of nSMase inhibitor (GW4869). Mitochondrial DNA copy number were quantified by assessing ND1 (mitochondrial gene) level which was normalized to HPRT (nuclear gene). Cellular ATP levels (C) and TG storage (D) were quantified after culturing C2C12 myotubes under similar conditions as described under 'Methods' section. Data are presented as mean + standard deviation. $n=4$, ${ }^{* *} P<0.001$, ${ }^{* *} P<0.01,{ }^{*} P<0.05$, one way ANOVA with Newman-Keuls post test was performed for statistical analyses. 

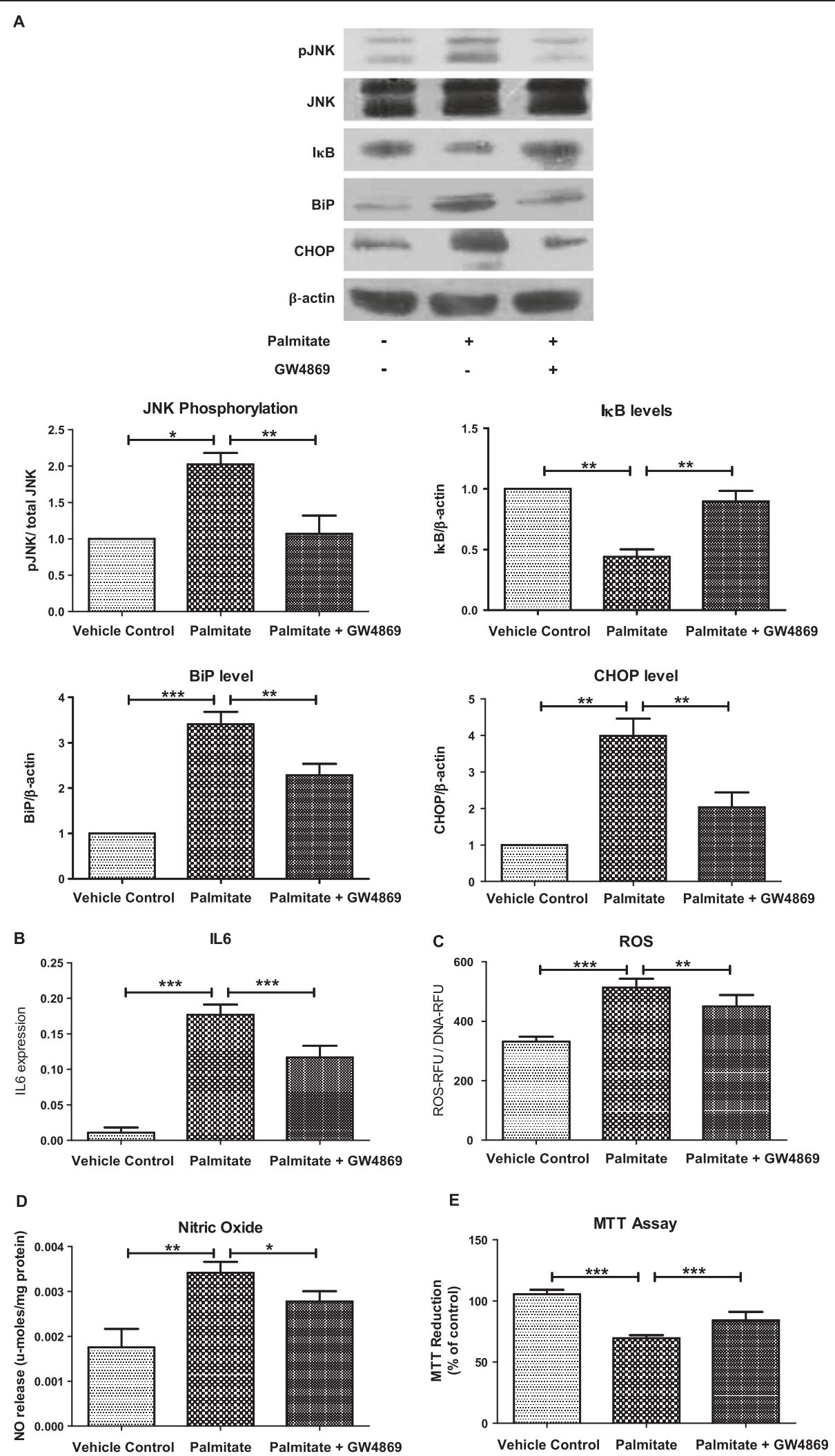

Figure 4 (See legend on next page.) 
(See figure on previous page.)

Figure 4 Reduction in stress signaling, inflammation, oxidative stress and ER stress by nSMase inhibition. (A) After palmitate treatment in presence or absence of nSMase inhibitor (GW4869), C2C12 myotubes were harvested in lysis buffer. Upper panel: Levels of phosphorylated JNK, unphosphorylated JNK, IKB, BiP and CHOP were measured using Western blotting. $\beta$-actin was used as a loading control. Lower panels: quantification of Western blotting data. (B) Gene expression of IL6 was measured by quantitative real time PCR using $\beta$-actin as housekeeping gene control. (C) After treatment, myotubes were loaded with DCFH-DA ROS indicator fluorescent probe to quantify the amount of cellular ROS. (D) Nitric oxide levels in culture medium were quantified using Griess reagent. (E) Myotubes viability was measured by MTT assay after treatment. Data are presented as mean + standard deviation. $n=4$, ${ }^{* *} \mathrm{P}<0.001$, ${ }^{* *} \mathrm{P}<0.01,{ }^{*} \mathrm{P}<0.05$, one way ANOVA with Newman-Keuls post test was performed for statistical analyses.

with unsaturated fatty acid (oleate) was unable to increase IL6 expression and inhibition of nSMase did not change it further (Additional file 2). Thus, nSMase inhibition can reduce palmitate induced stress signaling and inflammation.

Palmitate treatment resulted in enhanced BiP (3.4 fold of control, $\mathrm{P}<0.001$ ) and CHOP ( 4 fold of control, $\mathrm{P}<$ 0.01 ) levels demonstrating increased ER stress (Figure 4A). Inhibition of nSMase reduced ER stress as measured by partial reduction in the levels of $\mathrm{BiP}$ and $\mathrm{CHOP}(\mathrm{P}<0.01$, Figure 4A). Similarly, palmitate treatment elevated oxidative stress as levels of both reactive oxygen species (ROS) and reactive nitrite species (RNS) were found to be increased (Figure 4C, D). Both ROS and RNS levels were significantly reduced by nSMase inhibition (Figure 4C, D). Both ROS and RNS levels were unchanged under oleate treatment and nSMase inhibition did not show any effect under oleate (Additional file 2). Hence, palmitate induced both oxidative and ER stress are reduced by nSMase inhibitor.

Next, we analyzed the impact of palmitate treatment on myotubes survival. Palmitate treatment reduced cell survival as measured by MTT assay (70\% of vehicle control, $\mathrm{P}<0.001$, Figure $4 \mathrm{E})$. However, oleate treatment did not reduce cell survival (Additional file 2). Inhibition of nSMase under palmitate condition protected myotubes survival (84\% of control, $\mathrm{P}<0.001$, Figure $4 \mathrm{E}$ ). Taken together, nSMase inhibition reduces cellular stress and enhances cell survival under palmitate treatment.

\section{Discussion}

Lipid mediators are important signaling molecules for tissue homeostasis. Ceramides are potent lipid signaling molecules and act as metabolic hub because many other sphingolipids are derived from it (Scheffer et al. 2011; Pralhada Rao et al. 2013). Ceramide biology is becoming increasingly important because ceramide can impair activation of insulin signaling pathway and hence any modulation in its content can lead to better insulin sensitivity. In fact, a direct correlation exists between the concentration of saturated fatty acid present and the amount of ceramide generated (Adams et al. 2004). Also there is a direct correlation between amount of ceramide and degree of insulin resistance (Straczkowski et al. 2004, 2007; Turpin et al. 2006; Haus et al. 2009; Blachnio-Zabielska et al. 2012). Therefore, it is believed that generation of ceramide in response to FFA overload is one of the most important events causing insulin resistance (Schmitz-Peiffer et al. 1999).

FFA exposure initiates de novo synthesis of sphingolipids and concomitantly ceramide levels are also increased (Schmitz-Peiffer et al. 1999, Hu et al. 2009). Consequently, a number of groups tried to inhibit de novo synthesis of sphingolipids and reported better insulin sensitivity (Chavez et al. 2003; Powell et al. 2004; Holland et al. 2007; Watson et al. 2009; Ussher et al. 2010). However, it should be noted that not all species of sphingolipids can prompt insulin resistance. In fact, generation of sphingosine-1-phosphate (S1P) by sphingosine kinase (Hu et al. 2009) can ameliorate insulin resistance. S1P can increase glucose uptake in skeletal muscle cells (Rapizzi et al. 2009). Over-expression of sphingosine kinase to generate S1P or administration of S1P analog, FTY720, protect animal model of diabetes from insulin resistance (Kendall and Hupfeld 2008; Bruce et al. 2012, 2013). Moreover, conversion of ceramide to sphingosine by ceramidase hence providing substrate for S1P generation exerts negative correlation with FFA induced insulin resistance (Chavez et al. 2003, 2005). Hence, we sought to inhibit ceramide generation from sphingomyelin hydrolysis though the action of enzyme SMases which is found to be up-regulated during glucose intolerance (Murase et al. 1998; Straczkowski et al. 2007; Shah et al. 2008).

We exposed differentiated C2C12 myotubes for 16 hrs to saturated FFA palmitate which is known to increase cellular ceramide contents (Schmitz-Peiffer et al. 1999). To inhibit nSMases, we used a small molecule inhibitor, GW4869, which is reported to reduce ceramide levels (Luberto et al. 2002). Though we ourselves did not measure the total ceramide content, we observed an increase in expression of genes involved in ceramide synthesis upon palmitate treatment and estimated the functional outcomes after intervention in the ceramide synthesis pathway. To examine the relationship between nSMase and insulin resistance, we first demonstrated that the levels of all three nSMases were increased under palmitate with attendant decrease in insulin stimulated 
Akt/PKB phosphorylation. Thus our in vitro data are in agreement with what is observed in diabetic patients. Treatment with oleate did not increase the nSMases expression and it is reported that this fatty acid does not increase the ceramide content of the cells. Inhibition of nSMases, as expected, maintained insulin stimulated Akt phosphorylation indicating improved insulin sensitivity. Our data show that under nSMase inhibition, PDK4 expression was increased by palmitate and was decreased by insulin thus suggesting that metabolic flexibility of myotubes is maintained. PDK4 is a negative regulator of glucose oxidation which inhibits pyruvate entry into mitochondria for further oxidation while fat oxidation is promoted. Metabolic flexibility or ability to switch fuel utilization from fat to glucose is lost during insulin resistance since PDK4 levels are elevated (Kim et al. 2006, Tsintzas et al. 2007; Kulkarni et al. 2012). Insulin induced increase in Akt phosphorylation and decrease in PDK4 expression in myotubes cultured under nSMase inhibitor suggest functional insulin signaling cascade.

While a number of reports have authenticated connection between ceramide content and insulin resistant, we extended the study to see the effects on oxidative capacity given that overall oxidation is severely impaired in human patients (He et al. 2001; Oberbach et al. 2006; Koves et al. 2008). In order to study the impact on oxidative capacity we measured expression of PGC1 $\alpha$ - master regulator of mitochondrial biogenesis and oxidative capacity, mitochondrial number and ATP levels. All these indicators of oxidative capacity were reduced under palmitate whereas nSMase inhibition could significantly restore their levels. Improvement in oxidative capacity by nSMase inhibition also resulted in moderate but statistically significant reduction in TG storage. Only partial restoration in oxidative capacity and reduction in TG storage by nSMase inhibition might be a result of defects caused by saturated FFA other than ceramide synthesis or contribution of de novo ceramide biosynthesis as we observed an increase in expression of SPT, a rate limiting enzyme of this pathway.

Both saturated FFA and ceramide activate JNK and $\mathrm{NFKB}$ signaling which can lead to cellular stress (Itani et al. 2002; Yuzefovych et al. 2010; MohammadTaghvaei et al. 2012). Therefore we examined JNK and NFKB activation, oxidative and ER stress and generation of inflammatory cytokines. Consistent with the data on oxidative capacity, nSMase inhibition only partially reduced cellular stress and IL6 expression again indicating about role of FFA other than ceramide synthesis for cellular stress or contribution of de novo ceramide biosynthesis. Importance of de novo ceramide biosynthesis in this regard is already reported. One possible explanation about the role of FFA other than ceramide synthesis is that extracellular FFA can directly activate toll like receptors (TLRs) which downstream activate JNK and NFkB (Senn 2006). JNK and NFKB activation results in inflammatory cytokines production and elevation of cellular stress which itself can impair oxidative capacity and insulin signaling.

Overall our data establish, for the first time, the role of nSMase in protecting skeletal muscle from saturated FFA induced lipotoxic impacts while maintaining insulin sensitivity.

\section{Conclusions}

In this study, we report that inhibition of nSMase can prevent saturated FFA overload induced insulin resistance and multiple defects in metabolism. Inhibition of nSMase can also reduce palmitate induced stress and inflammation. Palmitate but not oleate treatment up-regulates expression of all three nSMases which generate ceramide from sphingomyelin. Palmitate treatment led to insulin resistance as well as to reduced oxidative capacity. Exposure of myotubes to palmitate caused oxidative stress, ER stress, stress signaling, inflammatory cytokine production and reduced myotubes survival. We show that these deleterious impacts of palmitate are largely by increased nSMase levels as inhibition of nSMase could revert these impacts (Figure 5). Hence, inhibition of nSMase can be a good approach to reduce skeletal muscle insulin resistance and defects in metabolism.

\section{Methods}

\section{Cell culture and palmitate treatment}

C2C12 myoblast cell line (ATCC) was maintained in undifferentiated state at low confluency in DMEM (Sigma) containing $25 \mathrm{mM}$ glucose, $10 \%$ fetal bovine serum (Invitrogen), penicillin and streptomycin. Myoblasts were differentiated into myotubes in multi-well plates after growing till complete confluency in growth medium and followed by culturing in reduced serum $(2 \%)$ medium for four days with media change at every alternate day. Fully differentiated cells evident by long multi-nucleated myotubes were used for the experiments.

Palmitate or oleate (Sigma) were dissolved in 50\% ethanol followed by conjugation with $10 \%$ BSA. Differentiated myotubes received BSA conjugated palmitate or oleate $(5 \mathrm{mM})$ to a final concentration of $750 \mu \mathrm{M}$ and were cultured for $16 \mathrm{hrs}$. Vehicle control treatment included equal amount of BSA and ethanol for $16 \mathrm{hrs.} \mathrm{For}$ inhibition of nSMases, GW4869 (Sigma) was also added to a final concentration of $10 \mu \mathrm{M}$ along with palmitate or oleate for $16 \mathrm{hrs}$.

\section{Analysis of gene expression}

Differentiated myotubes were cultured with indicated treatment for $16 \mathrm{hrs}$. After $16 \mathrm{hrs}$ of treatment, total RNA was extracted using Trizol reagent (Invitrogen) and 


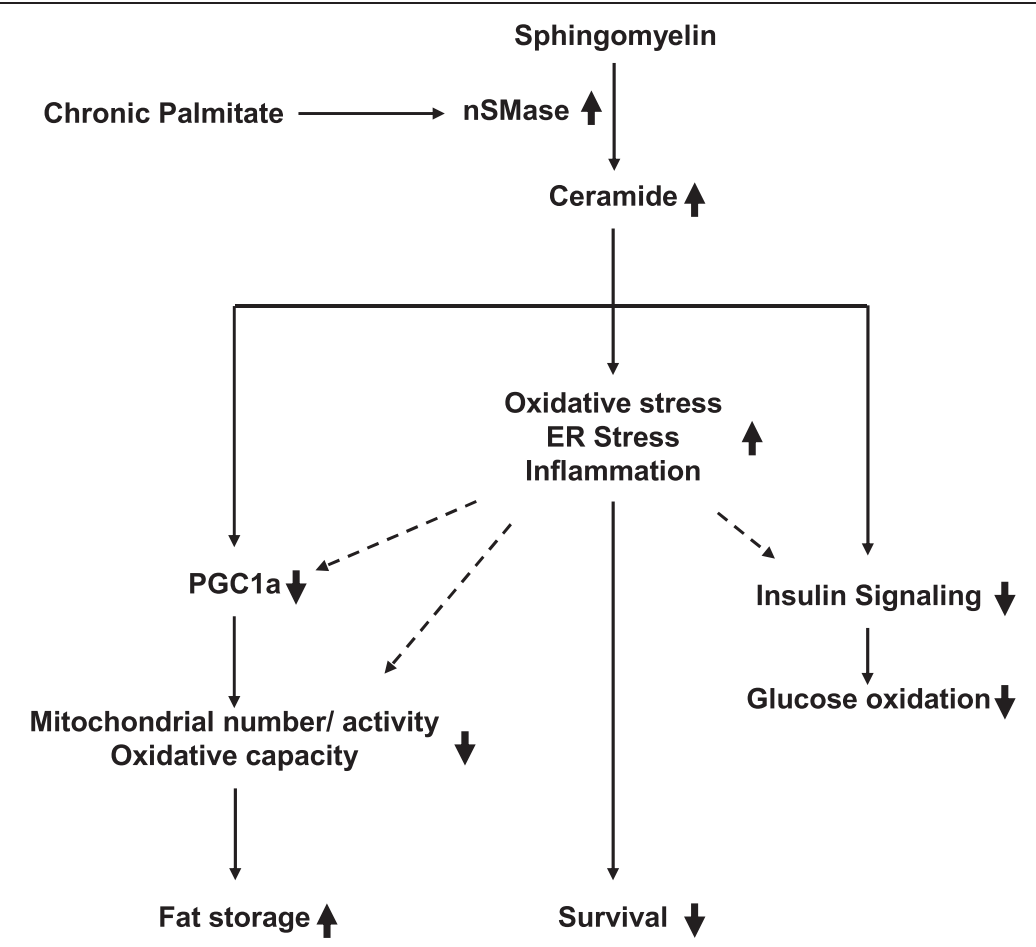

Figure $\mathbf{5}$ Saturated FFA mediated impairment in skeletal muscle and its reversal by nSMase inhibition. Sphingomyelin can be converted into ceramide by nSMases. Palmitate mediated up-regulation of nSMases can expedite this process of ceramide generation. Increased ceramide can lead to an inhibition on insulin signaling pathway thereby reducing glucose oxidation. In this condition PGC1a levels are reduced with a concomitant decrease in muscle oxidative capacity. Because of impaired oxidative function and palmitate overload, fat storage in form of TG is increased. Palmitate overload mediated increased ceramide levels elevate cellular oxidative stress, ER stress and inflammation which can severely affect cell survival. Elevated stress and inflammation can directly inhibit insulin signaling and can impair oxidative capacity (represented as dashed arrows). Palmitate mediated impact on cellular responses is represented by a thick up or down arrow placed just after particular cellular response. Inhibition of nSMase under this background reverses palmitate mediated impact on cellular response.

converted into cDNA with reverse transcriptase and random hexamer primers (Applied Biosystems). A total of 5 ng of cDNA was used for real time PCR for quantification of gene expression using SYBR Green PCR Master Mix (Eurogenetic). Genes analyzed in this study were nSMase1, nSMase-2, nSMase-3, SPTLC1, PDK4, PGC1 $\alpha$ and IL6. $\beta$-actin was used as a housekeeping gene control.

For PDK4 (pyruvate dehydrogenase kinase 4) expression, $\mathrm{C} 2 \mathrm{C} 12$ cells were cultured for $2 \mathrm{hrs}$ in presence or absence of vehicle, palmitate $(750 \mu \mathrm{M})$, oleate $(750 \mu \mathrm{M})$, GW4869 $(10 \mu \mathrm{M})$ or insulin $(100 \mathrm{nM})$. Cells were then harvested for gene expression analysis.

\section{Western blotting}

After treatment myotubes were lysed and amounts of total proteins were estimated using Bradford reagent (Bio-Rad). Equal amount of protein $(30 \mu \mathrm{g})$ was resolved by SDS-PAGE and then transferred to nitrocellulose membrane and signals were developed by chemiluminescence. Primary antibodies used were phospho-Akt, Akt, phospho-JNK, JNK, BiP, CHOP, $\beta$-actin (Cell Signaling Technology) and IкB (Abcam).
For the estimation of insulin sensitivity by Akt phosphorylation, myotubes were cultured under vehicle control or palmitate treatment in the presence or absence of GW4869 and then incubated in serum and glucose free medium for $30 \mathrm{~min}$. Subsequently, the myotubes were treated with insulin $(100 \mathrm{nM})$ for $10 \mathrm{~min}$ and were then harvested for western blotting.

Western blot bands were quantified by densitometric analysis using Image-J software (NIH).

\section{Estimation of mitochondrial DNA copy number}

Post 16 hrs of culture under indicated conditions, total DNA was isolated from myotubes. This total DNA (5 ng) was used for quantitative real time PCR using a primer pair specific to mitochondrial DNA (ND1) and a primer pair specific to nuclear DNA (HPRT). Mitochondrial DNA copy number was normalized to nuclear DNA.

\section{Estimation of cellular ATP levels}

After 16 hrs of culture, myotubes were harvested for estimation of cellular ATP content using commercially available kit (ATP determination kit, Invitrogen) as per 
manufacturer's protocol. Amount of ATP was normalized to total cellular protein.

\section{Estimation of triglyceride levels}

After culture, myotubes were lysed and clear lysate was used for TG estimation using a commercially available kit (DiaSys). TG levels were normalized to cellular proteins measured using Bradford reagent (Bio-Rad).

\section{Estimation of ROS}

After treatments for 16 hrs, myotubes were loaded with DCFH-DA dye (Invitrogen) for $1 \mathrm{~h}$ to measure ROS levels. Amount of ROS was normalized to total cellular DNA which was measured using bis-benzamide (Sigma). Bis-benzamide was added for $15 \mathrm{~min}$. Myotubes were lysed and lysates were transferred to 96-well black well plate. DCF fluorescence was measured at $485 \mathrm{~nm}$ excitation and $528 \mathrm{~nm}$ emission. DNA levels were measured at $360 \mathrm{~nm}$ excitation and $460 \mathrm{~nm}$ emission. Data are represented as ROS fluorescence (RFU)/DNA fluorescence (RFU).

\section{Estimation of nitric oxide release}

Nitric oxide release was measured in cell culture medium. After treatment, culture medium was centrifuged to remove any debris. Culture medium was mixed with equal amount of Griess reagent (Sigma) and incubated for $15 \mathrm{~min}$ followed by absorption measurement at $540 \mathrm{~nm}$. Amount of nitric oxide release was quantified by a standard curve prepared using sodium nitrite and were normalized to total cellular proteins measured using Bradford reagent (Bio-Rad).

\section{MTT assay}

After treatment, MTT dye was added to myotubes and incubated for $3 \mathrm{~h}$. After incubation culture medium was discarded and DMSO was added to each well to dissolve the reduced form of MTT (formazan). Amount of MTT reduced was estimated by $560 \mathrm{~nm}$ absorbance after correction with $640 \mathrm{~nm}$ absorbance. Reduction of MTT into formazan was represented as \% of vehicle control.

\section{Statistical analysis}

Data are represented as mean with standard deviation or mean with standard error of mean. Statistical significances were calculated by one way ANOVA with Newman-Keuls post test using GraphPad Prism. P-values were calculated between groups as indicated in figures and were represented as ${ }^{*} \mathrm{P}<0.05,{ }^{* *} \mathrm{P}<0.01$ and ${ }^{* * *} \mathrm{P}<0.001$.

\section{Additional files}

\begin{abstract}
Additional file 1: Impact of oleate treatment, nSMase inhibition and insulin on PDK4 expression. C2C12 cells were treated with or without oleate for 2 hrs in presence or absence of nSMase inhibitor (GW4869). Insulin was also present in the treatments for indicated sets. Gene expression level of PDK4 was analyzed by quantitative real time PCR using $\beta$-actin as housekeeping gene control. In vehicle control treated cells, insulin significantly inhibited PDK4 expression. Though oleate treatment increased the PDK4 expression, it did not cause insulin resistance as insulin was able to reduce the expression of PDK4. Inhibition of nSMase by GW4869 maintained the insulin sensitivity of C2C12 cells. Data are presented as mean + standard deviation. $n=4$, ${ }^{* *} P<0.01$, ${ }^{*} \mathrm{P}<0.05$, one way ANOVA with Newman-Keuls post test was performed for statistical analyses.
\end{abstract}

Additional file 2: Oleate treatment does not show any impact on inflammation, oxidative stress and cell survival. Myotubes were treated for 16 hrs either with vehicle control or with oleate $(750 \mu \mathrm{M})$ in presence or absence of GW4869. Gene expression of IL6 was measured by quantitative real time PCR using $\beta$-actin as housekeeping gene control (A). After treatment, myotubes were loaded with DCFH-DA ROS indicator fluorescent probe to quantify the amount of cellular ROS (B) and nitric oxide levels in culture medium were quantified using Griess reagent (C). Myotubes viability was measured by MTT assay (D). Data are presented as mean + standard deviation. $n=4$, one way ANOVA with Newman-Keuls post test was performed for statistical analyses and no statistical significance was observed among treatments indicating oleate treatment did not cause inflammation and cellular stress and did not impact viability.

\section{Abbreviations}

SMase: Sphingomyelinase; nSMase: Neutral SMase; SPT: Serine palmitoyl transferase; S1P: Sphingosine-1-phosphate; T2DM: Type-II Diabetes mellitus; FFA: Free fatty acid; TG: Triglyceride; ER: Endoplasmic reticulum; PDK4: Pyruvate dehydrogenase kinase 4; PGC1a: Peroxisome proliferatoractivated receptor gamma, Coactivator 1 Alpha; IL6: Interleukin 6; ROS: Reactive oxygen species.

\section{Competing interests}

The authors declare that they have no competing interest.

\section{Authors' contributions}

ANY, KN, NP, KS, JP, MNL, PMP and SY carried out the experiments. MKV conceived the idea, planned the study, analyzed the data and wrote the paper. AMO, BPS and MRJ contributed in planning the experiments and reviewing/editing the manuscript. All authors read and approved the final manuscript.

\section{Acknowledgements}

The authors thank Smitha R and Dr. Raghavendra Rao for their help in conducting the study, critical review of the manuscript and helpful discussions. We also thank Mrudula S, Naved Pasha, Prathibha R, Sowmya TS and Bhawna Chandravanshi at Connexios Life Sciences Pvt Ltd. for their assistance in the study.

Received: 17 January 2014 Accepted: 12 May 2014

Published: 20 May 2014

\section{References}

Adams JM 2nd, Pratipanawatr T, Berria R, Wang E, DeFronzo RA, Sullards MC, Mandarino $L J$ (2004) Ceramide content is increased in skeletal muscle from obese insulin-resistant humans. Diabetes 53:25-31

Asmann YW, Stump CS, Short KR, Coenen-Schimke JM, Guo Z, Bigelow ML, Nair KS (2006) Skeletal muscle mitochondrial functions, mitochondrial DNA copy numbers, and gene transcript profiles in type 2 diabetic and nondiabetic subjects at equal levels of low or high insulin and euglycemia. Diabetes 55:3309-3319

Baron AD, Brechtel G, Wallace P, Edelman SV (1988) Rates and tissue sites of non-insulin- and insulin-mediated glucose uptake in humans. Am J Physiol 255:E769-E774 
Blachnio-Zabielska A, Baranowski M, Zabielski P, Gorski J (2010) Effect of high fat diet enriched with unsaturated and diet rich in saturated fatty acids on sphingolipid metabolism in rat skeletal muscle. J Cell Physiol 225:786-791

Blachnio-Zabielska AU, Koutsari C, Tchkonia T, Jensen MD (2012) Sphingolipid content of human adipose tissue: relationship to adiponectin and insulin resistance. Obesity (Silver Spring) 20:2341-2347

Bonen A, Parolin ML, Steinberg GR, Calles-Escandon J, Tandon NN, Glatz JF, Luiken JJ, Heigenhauser GJ, Dyck DJ (2004) Triacylglycerol accumulation in human obesity and type 2 diabetes is associated with increased rates of skeletal muscle fatty acid transport and increased sarcolemmal FAT/CD36. FASEB J 18:1144-1146

Boon J, Hoy AJ, Stark R, Brown RD, Meex RC, Henstridge DC, Schenk S, Meikle PJ, Horowitz JF, Kingwell BA, Bruce CR, Watt MJ (2013) Ceramides contained in $\mathrm{LDL}$ are elevated in type 2 diabetes and promote inflammation and skeletal muscleinsulin resistance. Diabetes 62:401-110

Bouzakri K, Roques M, Gual P, Espinosa S, Guebre-Egziabher F, Riou JP, Laville M, Le Marchand-Brustel Y, Tanti JF, Vidal H (2003) Reduced activation of phosphatidylinositol-3 kinase and increased serine 636 phosphorylation of insulin receptor substrate-1 in primary culture of skeletal muscle cells from patients with type 2 diabetes. Diabetes 52:1319-1325

Bruce CR, Risis S, Babb JR, Yang C, Kowalski GM, Selathurai A, Lee-Young RS, Weir JM, Yoshioka K, Takuwa Y, Meikle PJ, Pitson SM, Febbraio MA (2012) Overexpression of sphingosine kinase 1 prevents ceramide accumulation and ameliorates muscle insulinresistance in high-fat diet-fed mice. Diabetes 61:3148-3155

Bruce CR, Risis S, Babb JR, Yang C, Lee-Young RS, Henstridge DC, Febbraio MA (2013) The sphingosine-1-phosphate analog FTY720 reduces muscle ceramide content and improves glucose tolerance in high fat-fed male mice. Endocrinology 154:65-76

Chavez JA, Knotts TA, Wang LP, Li G, Dobrowsky RT, Florant GL, Summers SA (2003) A role for ceramide, but not diacylglycerol, in the antagonism of insulin signal transduction by saturated fatty acids. J Biol Chem 278:10297-10303

Chavez JA, Holland WL, Bär J, Sandhoff K, Summers SA (2005) Acid ceramidase overexpression prevents the inhibitory effects of saturated fatty acids on insulin signaling. J Biol Chem 280:20148-20153

De Mello VD, Lankinen M, Schwab U, Kolehmainen M, Lehto S, Seppänen-Laakso T, Oresic M, Pulkkinen L, Uusitupa M, Erkkilä AT (2009) Link between plasma ceramides, inflammation and insulin resistance: association with serum IL-6concentration in patients with coronary heart disease. Diabetologia 52:2612-2615

Dobrzyń A, Górski J, Ceramides and sphingomyelins in skeletal muscles of the rat: content and composition (2002) Effect of prolonged exercise. Am J Physiol Endocrinol Metab 282:E277-E285

Goodpaster BH, Theriault R, Watkins SC, Kelley DE (2000) Intramuscular lipid content is increased in obesity and decreased by weight loss. Metabolism 49:467-472

Goodpaster BH, He J, Watkins S, Kelley DE (2001) Skeletal muscle lipid content and insulin resistance: evidence for a paradox in endurance-trained athletes. J Clin Endocrinol Metab 86:5755-5761

Haus JM, Kashyap SR, Kasumov T, Zhang R, Kelly KR, Defronzo RA, Kirwan JP (2009) Plasma ceramides are elevated in obese subjects with type 2 diabetes and correlate with the severity ofinsulin resistance. Diabetes 58:337-343

He J, Watkins S, Kelley DE (2001) Skeletal muscle lipid content and oxidative enzyme activity in relation to muscle fiber type in type 2 diabetes and obesity. Diabetes 50:817-823

Helge JW, Dobrzyn A, Saltin B, Gorski J (2004) Exercise and training effects on ceramide metabolism in human skeletal muscle. Exp Physiol 89:119-127

Hey-Mogensen M, Højlund K, Vind BF, Wang L, Dela F, Beck-Nielsen H, Fernström $M$, Sahlin K (2010) Effect of physical training on mitochondrial respiration and reactive oxygen species release in skeletal muscle in patients with obesity and type 2 diabetes. Diabetologia 53:1976-1985

Holland WL, Brozinick JT, Wang LP, Hawkins ED, Sargent KM, Liu Y, Narra K, Hoehn KL, Knotts TA, Siesky A, Nelson DH, Karathanasis SK, Fontenot GK, Birnbaum MJ, Summers SA (2007) Inhibition of ceramide synthesis ameliorates glucocorticoid-, saturated-fat-, and obesity-induced insulinresistance. Cell Metab 5:167-179

Hu W, Bielawski J, Samad F, Merrill AH Jr, Cowart LA (2009) Palmitate increases sphingosine-1-phosphate in C2C12 myotubes via upregulation of sphingosine kinase message and activity. J Lipid Res 50:1852-1862
Itani SI, Ruderman NB, Schmieder F, Boden G (2002) Lipid-induced insulin resistance in human muscle is associated with changes in diacylglycerol, protein kinase C, and IkappaB-alpha. Diabetes 51:2005-2011

Kelley DE, Mintun MA, Watkins SC, Simoneau JA, Jadali F, Fredrickson A, Beattie J, Thériault R (1996) The effect of non-insulin-dependent diabetes mellitus and obesity on glucose transport and phosphorylation in skeletal muscle. J Clin Invest 97:2705-2713

Kelley DE, He J, Menshikova EV, Ritov VB (2002) Dysfunction of mitochondria in human skeletal muscle in type 2 diabetes. Diabetes 51:2944-2950

Kendall MR, Hupfeld CJ (2008) FTY720, a sphingosine-1-phosphate receptor modulator, reverses high-fat diet-induced weight gain, insulinresistance and adipose tissue inflammation in C57BL/6 mice. Diabetes Obes Metab 10:802-805

Kim YB, Nikoulina SE, Ciaraldi TP, Henry RR, Kahn BB (1999) Normal insulindependent activation of Akt/protein kinase $B$, with diminished activation of phosphoinositide 3-kinase, in muscle in type 2 diabetes. J Clin Invest 104:733-741

Kim Yl, Lee FN, Choi WS, Lee S, Youn JH (2006) Insulin regulation of skeletal muscle PDK4 mRNA expression is impaired in acute insulin-resistant states. Diabetes 55:2311-2317

Koves TR, Ussher JR, Noland RC, Slentz D, Mosedale M, Ilkayeva O, Bain J, Stevens R, Dyck JR, Newgard CB, Lopaschuk GD, Muoio DM (2008) Mitochondrial overload and incomplete fatty acid oxidation contribute to skeletal muscle insulin resistance. Cell Metab 7:45-56

Krook A, Björnholm M, Galuska D, Jiang XJ, Fahlman R, Myers MG Jr, WallbergHenriksson H, Zierath JR (2000) Characterization of signal transduction and glucose transport in skeletal muscle from type 2 diabetic patients. Diabetes 49:284-292

Kulkarni SS, Salehzadeh F, Fritz T, Zierath JR, Krook A, Osler ME (2012) Mitochondrial regulators of fatty acid metabolism reflect metabolic dysfunction in type 2 diabetes mellitus. Metabolism 61:175-185

Listenberger LL, Han X, Lewis SE, Cases S, Farese RV Jr, Ory DS, Schaffer JE (2003) Triglyceride accumulation protects against fatty acid-induced lipotoxicity. Proc Natl Acad Sci U S A 100:3077-3082

Long SD, Pekala PH (1996) Lipid mediators of insulin resistance: ceramide signalling down-regulates GLUT4 gene transcription in 3T3-L1 adipocytes. Biochem J 319:179-184

Luberto C, Hassler DF, Signorelli P, Okamoto Y, Sawai H, Boros E, Hazen-Martin DJ, Obeid LM, Hannun YA, Smith GK (2002) Inhibition of tumor necrosis factor-induced cell death in MCF7 by a novel inhibitor of neutral sphingomyelinase. J Biol Chem 277:41128-41139

McGarry JD (2002) Banting lecture 2001: dysregulation of fatty acid metabolism in the etiology of type 2 diabetes. Diabetes 51:7-18

Mensink M, Hesselink MK, Russell AP, Schaart G, Sels JP, Schrauwen P (2007) Improved skeletal muscle oxidative enzyme activity and restoration of PGC-1 alpha and PPAR beta/delta gene expression upon rosiglitazone treatment in obese patients with type 2 diabetes mellitus. Int J Obes (Lond) 31:1302-1310

Mogensen M, Sahlin K, Fernström M, Glintborg D, Vind BF, Beck-Nielsen H, Højlund K (2007) Mitochondrial respiration is decreased in skeletal muscle of patients with type 2 diabetes. Diabetes 56:1592-1599

MohammadTaghvaei N, Taheripak G, Taghikhani M, Meshkani R (2012) Palmitateinduced PTP1B expression is mediated by ceramide-JNK and nuclear factor KB (NF-KB) activation. Cell Signal 24:1964-1970

Mootha VK, Lindgren CM, Eriksson KF, Subramanian A, Sihag S, Lehar J, Puigserver $P$, Carlsson E, Ridderstråle M, Laurila $E$, Houstis N, Daly MJ, Patterson N, Mesirov JP, Golub TR, Tamayo P, Spiegelman B, Lander ES, Hirschhorn JN, Altshuler D, Groop LC (2003) PGC-1alpha-responsive genes involved in oxidative phosphorylation are coordinately downregulated in human diabetes. Nat Genet 34:267-273

Murase K, Odaka H, Suzuki M, Tayuki N, Ikeda H (1998) Pioglitazone timedependently reduces tumour necrosis factor-alpha level in muscle and improves metabolic abnormalities in Wistar fatty rats. Diabetologia 41:257-264

Oberbach A, Bossenz Y, Lehmann S, Niebauer J, Adams V, Paschke R, Schön MR, Blüher M, Punkt K (2006) Altered fiber distribution and fiber-specific glycolytic and oxidative enzyme activity in skeletal muscle of patients with type 2 diabetes. Diabetes Care 29:895-900

Patti ME, Butte AJ, Crunkhorn S, Cusi K, Berria R, Kashyap S, Miyazaki Y, Kohane I, Costello M, Saccone R, Landaker EJ, Goldfine AB, Mun E, DeFronzo R, Finlayson J, Kahn CR, Mandarino LJ (2003) Coordinated reduction of genes of oxidative metabolism in humans with insulin resistance and diabetes: Potential role of PGC1 and NRF1. Proc Natl Acad Sci U S A 100:8466-8471 
Pickersgill L, Litherland GJ, Greenberg AS, Walker M, Yeaman SJ (2007) Key role for ceramides in mediating insulin resistance in human muscle cells. J Biol Chem 282:12583-9

Powell DJ, Turban S, Gray A, Hajduch E, Hundal HS (2004) Intracellular ceramide synthesis and protein kinase Czeta activation play an essential role in palmitate-induced insulin resistance in rat L6 skeletal muscle cells. Biochem J 382:619-629

Pralhada Rao R, Vaidyanathan N, Rengasamy M, Mammen Oommen A, Somaiya N, Jagannath MR (2013) Sphingolipid metabolic pathway: an overview of major roles played in human diseases. J Lipids 2013:178910

Rapizzi E, Taddei ML, Fiaschi T, Donati C, Bruni P, Chiarugi P (2009) Sphingosine 1-phosphate increases glucose uptake through trans-activation of insulin receptor. Cell Mol Life Sci 66:3207-3218

Scheffer L, Raghavendra PR, Ma J, Acharya JK (2011) Ceramide transfer protein and cancer. Anticancer Agents Med Chem 11:904-910

Scheuermann-Freestone M, Madsen PL, Manners D, Blamire AM, Buckingham RE, Styles P, Radda GK, Neubauer S, Clarke K (2003) Abnormal cardiac and skeletal muscle energy metabolism in patients with type 2 diabetes. Circulation 107:3040-3046

Schmitz-Peiffer C, Craig DL, Biden TJ (1999) Ceramide generation is sufficient to account for the inhibition of the insulin-stimulated PKB pathway inC2C12 skeletal muscle cells pretreated with palmitate. J Biol Chem 274:24202-24210

Schrauwen P, Hesselink MK (2004) Oxidative capacity, lipotoxicity, and mitochondrial damage in type 2 diabetes. Diabetes 53:1412-1417

Senn JJ (2006) Toll-like receptor-2 is essential for the development of palmitateinduced insulin resistance in myotubes. J Biol Chem 281:26865-26875

Shah C, Yang G, Lee I, Bielawski J, Hannun YA, Samad F (2008) Protection from high fat diet-induced increase in ceramide in mice lacking plasminogen activator inhibitor 1. J Biol Chem 283:13538-13548

Simoneau JA, Kelley DE (1997) Altered glycolytic and oxidative capacities of skeletal muscle contribute to insulin resistance in NIDDM. J Appl Physiol (1985) 83:166-171

Straczkowski M, Kowalska I, Nikolajuk A, Dzienis-Straczkowska S, Kinalska I, Baranowski M, Zendzian-Piotrowska M, Brzezinska Z, Gorski J (2004) Relationship between insulin sensitivity and sphingomyelin signaling pathway in human skeletal muscle. Diabetes 53:1215-1221

Straczkowski M, Kowalska I, Baranowski M, Nikolajuk A, Otziomek E, Zabielski P, Adamska A, Blachnio A, Gorski J, Gorska M (2007) Increased skeletal muscle ceramide level in men at risk of developing type 2 diabetes. Diabetologia 50:2366-2373

Szendroedi J, Schmid Al, Chmelik M, Toth C, Brehm A, Krssak M, Nowotny P, Wolzt M, WaldhausI W, Roden M (2007) Muscle mitochondrial ATP synthesis and glucose transport/phosphorylation in type 2 diabetes. PLoS Med 4:e154

Tsintzas K, Chokkalingam K, Jewell K, Norton L, Macdonald IA, ConstantinTeodosiu D (2007) Elevated free fatty acids attenuate the insulin-induced suppression of PDK4 gene expression in human skeletal muscle: potential role of intramuscular long-chain acyl-coenzyme A. J Clin Endocrinol Metab 92:3967-3972

Turpin SM, Lancaster GI, Darby I, Febbraio MA, Watt MJ (2006) Apoptosis in skeletal muscle myotubes is induced by ceramides and is positively related to insulinresistance. Am J Physiol Endocrinol Metab 291:E1341-E1350

Ussher JR, Koves TR, Cadete VJ, Zhang L, Jaswal JS, Swyrd SJ, Lopaschuk DG, Proctor SD, Keung W, Muoio DM, Lopaschuk GD (2010) Inhibition of de novo ceramide synthesis reverses diet-induced insulin resistance and enhances whole-bodyoxygen consumption. Diabetes 59:2453-2464

van der Vusse GJ, Reneman RS (1996) Lipid metabolism in muscle. In: Handbook of Physiology: Exercise: Regulation and Integration of Multiple Systems. Am. Physiol. Soc., sect. 12, chapt. 21, Bethesda, MD, pp 952-994

Watson ML, Coghlan M, Hundal HS (2009) Modulating serine palmitoyl transferase (SPT) expression and activity unveils a crucial role in lipid-inducedinsulin resistance in rat skeletal muscle cells. Biochem J 417:791-801

Yang G, Badeanlou L, Bielawski J, Roberts AJ, Hannun YA, Samad F (2009) Central role of ceramide biosynthesis in body weight regulation, energy metabolism, and the metabolic syndrome. Am J Physiol Endocrinol Metab 297:E211-E224
Yuzefovych L, Wilson G, Rachek L (2010) Different effects of oleate vs. palmitate on mitochondrial function, apoptosis, and insulin signaling in L6skeletal muscle cells: role of oxidative stress. Am J Physiol Endocrinol Metab 299: E1096-E1105

Zierath JR (2007) The path to insulin resistance: paved with ceramides? Cell Metab 5:161-163

doi:10.1186/2193-1801-3-255

Cite this article as: Verma et al:: Inhibition of neutral sphingomyelinases in skeletal muscle attenuates fatty-acid induced defects in metabolism and stress. SpringerPlus 2014 3:255.

\section{Submit your manuscript to a SpringerOpen ${ }^{\circ}$ journal and benefit from:}

- Convenient online submission

- Rigorous peer review

- Immediate publication on acceptance

- Open access: articles freely available online

- High visibility within the field

- Retaining the copyright to your article

Submit your next manuscript at $>$ springeropen.com 